\title{
SECAGEM DE PASTA DE QUITOSANA EM CAMADA DELGADA: AVALIAÇÃO DO EFEITO DA SECAGEM SOBRE O GRAU DE DESACETILAÇÃO E A MASSA MOLECULAR VISCOSIMÉTRICA
}

\section{DRYING PASTE OF CHITOSAN IN THIN LAYER: EVALUATION OF THE EFFECT OF DRYING ON DEACETYLATION DEGREE AND VISCOSITY MOLECULAR WEIGHT}

\author{
Catarina Motta de Moura ${ }^{1}$, Jaqueline Motta de Moura ${ }^{2}$, Luiz Antonio de \\ Almeida Pinto ${ }^{3}$ \\ 1,2,3 Universidade Federal do Rio Grande - FURG, Laboratório de Operações Unitárias, \\ Escola de Química e Alimentos, Caixa Postal 474, 96201900 - Rio Grande, RS, \\ Brasil 3e-mail: dqmpinto@furg.br
}

\begin{abstract}
RESUMO
A quitosana é um biopolímero derivado da quitina, sendo que esta é encontrada na carapaça e exoesqueleto de crustáceos e outros materiais biológicos. A massa molecular e o grau de desacetilação deste biopolímero são características que determinam sua aplicação específica, como a produção de filme polimérico para revestimento de frutas e vegetais. A operação de secagem é uma importante etapa no processo de produção de quitosana, podendo, no entanto, ocorrer a polimerização do material durante esta operação. O objetivo deste trabalho foi determinar a massa molecular e o grau de desacetilação da quitosana obtida, utilizando diferentes condições de secagem em camada delgada. Os experimentos foram realizados em secador descontínuo de bandeja com escoamento paralelo do ar, nas temperaturas de 60 e $70^{\circ} \mathrm{C}$ e velocidade de $3 \mathrm{~m} / \mathrm{s}$. A quitosana foi seca na forma de pellets cilíndricos, em bandeja perfurada com $3 \mathrm{~mm}$ de espessura e carga de sólidos de $4 \mathrm{~kg} / \mathrm{m}^{2}$. A massa molecular e o grau de desacetilação foram determinados pelos métodos viscosimétrico e potenciométrico, respectivamente. A quitosana úmida apresentou massa molecular de $180 \mathrm{kDa}$ e $87 \%$ de grau de desacetilação. Os valores da massa molecular do produto final foram na faixa de $205 \mathrm{kDa}$; e não foram observadas alterações no grau de desacetilação após a secagem. O maior rendimento foi alcançado na temperatura de $70^{\circ} \mathrm{C}$.
\end{abstract}

Palavras-chave: Biopolímero. Quitina. Quitosana. Secagem de alimentos.

\begin{abstract}
This paper considers the use of Chitosan, a biopolymer obtained from chitin that is found in crustaceans exoskeletons and other biologic materials. The molecular weight and deacetylation degree of this biopolymer are the characteristics that determine its specific application as a polymeric film for fruit


and vegetable coating. The drying operation is an important step in production of chitosan, however the polymerization of the material can occur during this operation. The aim of this study was to determine the molecular weight and deacetylation degree of dried chitosan using different drying conditions in thin layers. The experiments were performed in discontinuous tray with parallel air flow dryer, at 60 and $70^{\circ} \mathrm{C}$ and air velocity at $3 \mathrm{~m} / \mathrm{s}$. The dried chitosan as cylindric pellets form was obtained in perforated tray with $3 \mathrm{~mm}$ thickness and solids loads in tray at $4 \mathrm{~kg} / \mathrm{m}^{2}$. The molecular weight and deacetylation degree were determined by viscosimetric and potentiometric methods, respectively. The wet chitosan presented molecular weight of $180 \mathrm{kDa}$ and the deacetylation degree of $87 \%$. The final product molecular weight values were on average $205 \mathrm{kDa}$, and did not show alterations in the degree of deacetylation after drying. The higher production of dried chitosan was determined at $70^{\circ} \mathrm{C}$.

Keywords: Biopolymer. Chitin. Chitosan. Food drying.

\section{Introdução}

A quitosana é considerada um alimento funcional aplicado em vários ramos da indústria alimentícia, apresentando aplicações também nas indústrias química e biomédica, bem como no tratamento de efluentes. A quitina e a quitosana são copolímeros constituídos, respectivamente, por unidades $\mathrm{N}$ acetil-D-glicosamina e D-glicosamina em proporções variáveis. A quitina é encontrada em abundância na natureza, como na carapaça e exoesqueleto de crustáceos, parede celular de fungos e em outros materiais biológicos. A quitosana pode ser obtida a partir da quitina por reação de desacetilação. (CRAVEIRO; CRAVEIRO; QUEIROZ, 1999; SILVA; SANTOS; FERREIRA, 2006).

O processo de produção de quitosana segue as etapas descritas por Weska et al. (2007) para a obtenção da quitina a partir de resíduos de camarão, a qual, após secagem, é desacetilada, produzindo-se a quitosana. Esta passa por um processo de purificação (ROBERTS; DOMSZY, 1982) e operação de secagem, geralmente realizada em secadores de bandejas. (BRZESKI, 1992).

De acordo com o grau médio de desacetilação, podem-se obter diversas quitosanas com diferentes propriedades físico-químicas, como solubilidade, viscosidade, biodegradabilidade, atividade imunológica (TOLAIMATE et al., 2003), assim como formação de géis, capacidade filmogênica e boas propriedades mecânicas. (ASSIS; SILVA, 2003). Quitosanas com grau de desacetilação acima da faixa de $65-70 \%$ são solúveis em ácidos orgânicos diluídos. (REGE; BLOCK, 1999).
Estudos realizados por Coma et al. (2002) mostram que filmes obtidos de polissacarídeos têm sido avaliados, com relativo sucesso, como coberturas semipermeáveis objetivando a extensão da vida útil de frutas e hortaliças, sendo a quitosana o composto mais estudado. Para a produção de filmes e membranas de quitosana, a operação de secagem é uma importante etapa do processo, pois nela pode ocorrer a polimerização do material, afetando as características do produto final.

O objetivo deste trabalho foi determinar a massa molecular, o grau de desacetilação e a produção da quitosana seca em camada delgada, com escoamento paralelo do ar, utilizando temperaturas do ar de secagem de 60 e $70^{\circ} \mathrm{C}$.

\section{Materais e métodos}

\subsection{Matéria-prima}

A fonte de extração da quitina e suas características influenciam diretamente na velocidade da reação de desacetilação. (CHANG et al., 1997). A quitina utilizada nos experimentos foi extraída a partir de resíduos de camarão-rosa (Farfantepenaeus brasiliensis).

A desacetilação da quitina para a produção de quitosana foi realizada utilizando-se solução alcalina concentrada $(42,7 \% \mathrm{~m} / \mathrm{v})$, à temperatura de $130^{\circ} \mathrm{C}$, durante 90 minutos, sob agitação constante de 60 rpm. (WESKA, et al. 2007). 
A purificação da quitosana foi processada valendo-se de procedimento análogo ao descrito por Robert; Domszy (1982), pelo qual esta foi dissolvida em solução ácida, centrifugada a $6600 \mathrm{~g}$, em centrífuga (modelo SIGMA 6-15, D-37520). O material não dissolvido foi precipitado com solução alcalina até $\mathrm{pH} 12,5$, neutralizado com solução ácida até pH 6,5 e centrifugado a $6600 \mathrm{~g}$, para a remoção do sobrenadante.

\subsection{Equipamento de secagem}

Utilizou-se secador de bandeja, com escoamento do ar paralelo às amostras. As bandejas eram de aço inoxidável, de forma retangular e perfuradas (mesh 9), mediam 0,2 $\mathrm{m}$ de largura, 0,25 $\mathrm{m}$ de comprimento e $3 \mathrm{~mm}$ de espessura

\subsection{Procedimento experimental}

As amostras foram preparadas a partir de pasta de quitosana úmida e convertidas em pellets cilíndricos. As bandejas foram pesadas, antes e depois de cheias, em balança eletrônica (modelo MARTE AS2000C) com precisão de $0,01 \mathrm{~g}$, e acondicionadas no secador.

A carga de pellets de quitosana utilizada foi de $4 \mathrm{~kg} / \mathrm{m}^{2}$, com temperaturas do ar de secagem de 60 e $70^{\circ} \mathrm{C}$ e umidade absoluta do ar de $0,022 \mathrm{~kg}$ vapor/ $\mathrm{kg}$ ar seco. Foram medidas as massas das amostras a cada 10 minutos. Todos os ensaios experimentais foram realizados em duplicata.

\subsection{Metodologia analítica}

O teor de umidade das amostras e do produto final foi determinado por secagem em estufa a $105^{\circ} \mathrm{C}$ por 24 horas, de acordo com a A.O.A.C. (1995).

\subsubsection{Determinação da massa molecular}

As amostras de quitosana foram dissolvidas em um sistema solvente constituído do ácido acético $0,1 \mathrm{M}$, do cloreto de sódio $0,2 \mathrm{M}$ e água. A viscosidade das amostras foi medida em um viscosímetro capilar Cannon-Fenske (Schott Geraete, modelo GMBH-D65719, Alemanha), em cinco diferentes concentrações (entre 0,1 e 1,2 g. $\mathrm{L}^{-1}$ ), a $25 \pm 1^{\circ} \mathrm{C}$.

No capilar foram colocados $10 \mathrm{~mL}$ de amostra, que passaram através dele, medindo-se o tempo de escoamento em segundos. O tempo de escoamento da solução e do solvente através do capilar foi utilizado para calcular a viscosidade cinemática. A relação entre a viscosidade cinemática e dinâmica é mostrada na Equação 1:

$$
v=\frac{\mu}{\rho}
$$

onde $v$ é a viscosidade cinemática (Stoke); $\mu$ é a viscosidade dinâmica (Poise); $\rho$ é a densidade da $\operatorname{amostra}(\mathrm{g} / \mathrm{mL})$.

A viscosidade específica $(\eta p)$ da solução e do solvente foi determinada utilizando-se a Equação 2:

$$
\eta p=\frac{\mu_{\text {solução }}-\mu_{\text {solvente }}}{\mu_{\text {solvente }}}
$$

A viscosidade intrínseca [ $\eta]$ foi estimada por ajuste da equação de Huggins de acordo com Simal (2002) (Equação 3):

$$
\frac{\eta \Phi}{c}=[\eta]+k \cdot[\eta]^{2} \cdot c
$$

onde $(\eta \mathrm{sp} / \mathrm{c})$ é a viscosidade reduzida em $\mathrm{mL} \cdot \mathrm{g}^{-1} ; \mathrm{c}$ é a concentração das soluções em $\mathrm{g} \cdot \mathrm{mL}^{-1}$; $\mathrm{k}$ é a constante válida para cada polímero em g. $\mathrm{mL}^{-1}$; $\mathrm{e}[\eta]$ é a viscosidade intrínseca em $\mathrm{mL} \cdot \mathrm{g}^{-1}$.

A viscosidade intrínseca [ $\eta]$ da solução foi determinada através de um gráfico da viscosidade reduzida $(\eta \mathrm{sp} / \mathrm{c})$ em função da concentração (c) das soluções das amostras, por extrapolação da reta obtida por regressão linear para $\mathrm{c}=0$, conforme apresentado por Alsarra et al. (2002).

A massa molecular viscosimétrica média $\left(\mathrm{M}_{\mathrm{v}}\right)$ da quitosana foi calculada a partir do valor da viscosidade intrínseca usando-se a equação empírica de Mark-Houwink-Sakurada (Equação 4), citada por Roberts \& Domszy (1982):

$$
[n]=k \cdot M_{V}^{\alpha}
$$




\section{2}

onde $\mathrm{k}=1,8 \times 10^{-3}$ e $\alpha=0,93$ são constantes que dependem do sistema solvente-polímero, determinadas por Roberts \& Domszy (1982), (GUPTA; JABRIAL, 2006; WESKA et al., 2007).

\subsubsection{Determinação do grau de desacetilação}

Para a determinação do grau de desacetilação foi utilizado o método de titulação potenciométrico linear (TAN et al, 1998; JIANG; CHEN; ZHONG, 2003), em que as amostras de quitosana foram dissolvidas em ácido clorídrico $(0,1 \mathrm{~N})$ e tituladas com hidróxido de sódio $(0,1 \mathrm{~N})$, nas faixas de $\mathrm{pH} 2,0$ a 6,0, em pHmetro (modelo MB10-Marte).

A curva de titulação linear foi obtida plotandose $\mathrm{f}(\mathrm{x})$ pelo volume correspondente de hidróxido de sódio. O volume de hidróxido de sódio no ponto de extremidade da titulação, Ve, foi estimado extrapolando-se a curva linear de titulação.

$O$ valor de $\mathrm{f}(\mathrm{x})$ do volume correspondente ao hidróxido de sódio adicionado foi calculado utilizando-se a Equação 5, mostrada a seguir:

$$
f(x)=\left(\frac{V_{0}+V}{N_{B}}\right) \cdot\left(\left[H^{+}\right]-\left[\theta^{-}\right]\right.
$$

onde Vo é o volume da solução inicial de quitosana (L); V é o volume do hidróxido de sódio adicionado (L); $\mathrm{N}_{\mathrm{B}}$ é a concentração de hidróxido de sódio $(\mathrm{N})$.

Para o cálculo do grau de desacetilação (GD\%) da amostra de quitosana aplicou-se a Equação 6, mostrada a seguir:

$$
\mathscr{\Theta}(\%)=\varnothing /[(W-161 \varnothing) / 204+\varnothing] \times 100
$$

onde ; $\mathrm{N}_{\mathrm{A}} \varnothing=\left(N_{A} V_{A}-N_{B} V_{e}\right) / 1000$ é a concentração de ácido clorídrico $(\mathrm{N}) ; \mathrm{V}_{\mathrm{A}}$ é o volume de ácido clorídrico $(\mathrm{mL}) ; \mathrm{N}_{\mathrm{B}}$ é a concentração de hidróxido de sódio $(\mathrm{N})$; Ve é o volume do hidróxido de sódio no ponto de extremidade $(\mathrm{mL})$; $\mathrm{W}$ é a massa da amostra $(\mathrm{g}) ; 161$ corresponde a massa molar da unidade D-glucosamina (mg. $\left.\mathrm{mol}^{-1}\right)$.

\subsubsection{Metodologia de cálculo}

Os valores da massa molecular e do grau de desacetilação, obtidos nos experimentos de secagem, foram comparados utilizando-se o teste de Tukey de diferenças de médias (Box, Hunter \& Hunter, 1978), considerando-se um nível de 0,05 de significância, com auxílio do programa Statistica 5.0.

Uma importante correlação na secagem de alimentos é a correlação exponencial. Esta correlação estabelece que a taxa de secagem é proporcional ao teor de água livre do gênero alimentício. É normalmente utilizada na forma integrada com dois parâmetros, conforme apresentada na Equação 7. (CHIRIFE, 1983):

$$
Y=\left(\frac{\overline{\mathrm{X}}-\mathrm{X}_{\mathrm{E}}}{\mathrm{X}_{\mathrm{o}}-\mathrm{X}_{\mathrm{E}}}\right)=A \cdot \exp \left(-\mathrm{K}_{1,2}\right)
$$

onde Y é o adimensional de água livre; A é um parâmetro de ajuste (adimensional); t é o tempo de secagem (min); e $\mathrm{K}_{1,2}$ é a constante de secagem $\left(\min ^{-1}\right)$, que é função das condições externas do ar (temperatura, velocidade e umidade) e das condições internas do material (espessura e umidade inicial).

O ajuste da Equação 7 aos dados experimentais da secagem de quitosana foi avaliado pelo coeficiente de determinação $\left(\mathrm{R}^{2}\right)$ e pelo erro médio relativo $(\mathrm{E} \%)$, que é definido pela Equação 8:

$$
E \%=\frac{1}{m}\left(\sum_{i=1}^{m} \frac{\left|Y_{\dot{\boldsymbol{e}}}-Y_{\dot{p}}\right|}{Y p i}\right) .100
$$

onde $\mathrm{Y}_{\mathrm{ei}}$ é o adimensional de água livre experimental; $\mathrm{Y}_{\mathrm{pi}}$ é o adimensional de água livre predito pelo ajuste da Equação 7; e m é o número de pontos experimentais.

A taxa de secagem $(\mathrm{N})$ foi obtida a partir da Equação 9:

$$
\mathrm{N}=\left(\frac{\mathbb{X}}{\mathrm{t}}\right) \cdot \frac{\mathrm{m}_{\mathrm{s}}}{\mathrm{S}}
$$

onde (dX/dt) é a velocidade de secagem ( $\mathrm{kg}$ água/kgss.min); $\mathrm{S}$ é a área de secagem; e $\mathrm{m}_{\mathrm{ss}}$ a massa de sólido na bandeja. 


\section{Resultados e discussão}

\subsection{Caracterização da Secagem em Camada Delgada}

A quitosana apresentou umidade inicial (x) igual a $93,1 \%$ em base úmida, o que corresponde a $13,5 \mathrm{~kg}$ água/ kgss (em base seca).

As Figuras 1 e 2 mostram as curvas de secagem do adimensional de umidade em função do tempo e da taxa de secagem em função da umidade dos pellets de quitosana, obtidas para 60 e $70^{\circ} \mathrm{C}$, respectivamente.

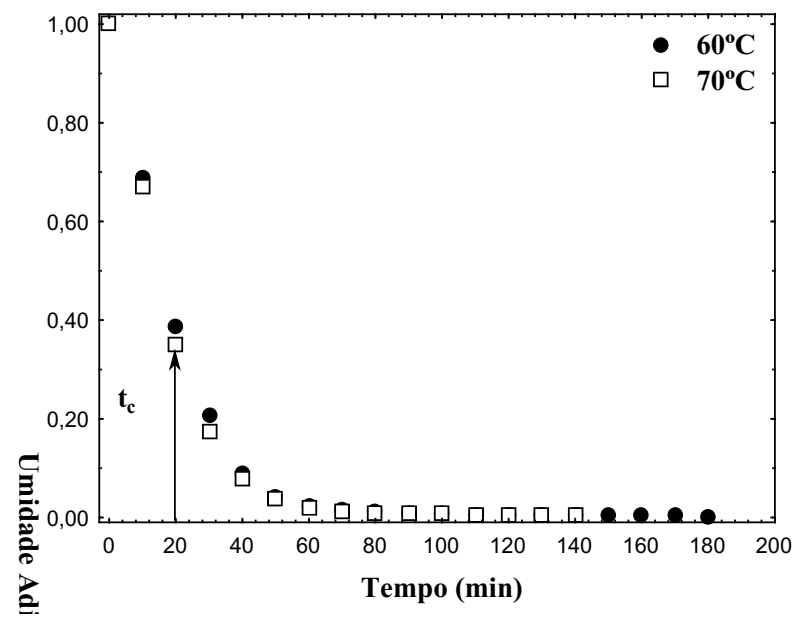

Figura 1: Curva de secagem do adimensional de umidade da quitosana em função do tempo de secagem, para 60 e $70^{\circ} \mathrm{C}$.

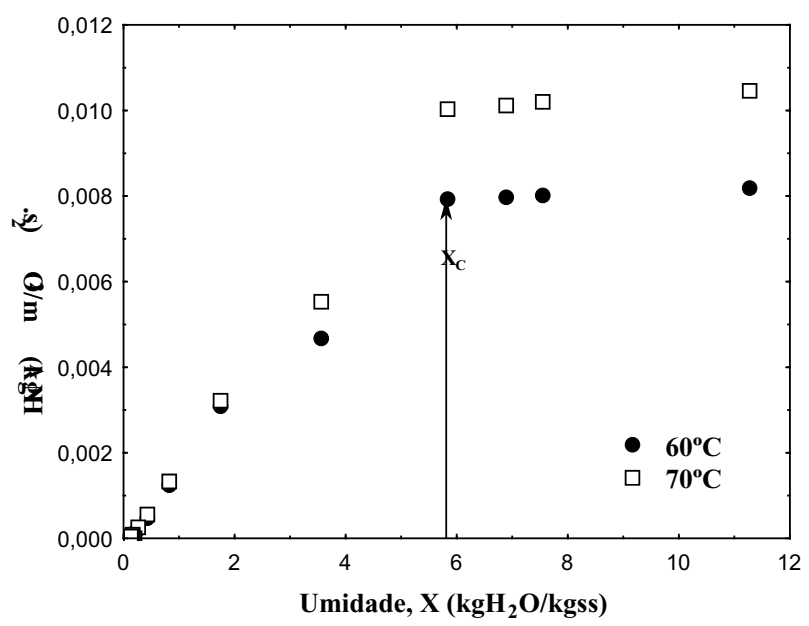

Figura 2: Curva da taxa de secagem em função da umidade da quitosana, para 60 e $70^{\circ} \mathrm{C}$.

Analisando as curvas de secagem, pode-se constatar nas Figuras 1 e 2, a existência do período de taxa constante, devido à alta umidade inicial do material. (CHIRIFE, 1983). Na Figura 1 observa-se que este período apresentou uma pequena duração (20min) em relação ao tempo total de secagem. Os valores da umidade crítica $\left(\mathrm{X}_{\mathrm{C}}\right)$, ao final do período de taxa constante mostrado na Figura 2, foi de 5,8 (b.s) em ambas as temperaturas de secagem utilizadas. Estes valores foram similares aos encontrados por Batista, Rosa \& Pinto (2007), que secaram quitosana na forma de pasta.

A Figura 3 apresenta as curvas do adimensional de água livre em função do tempo nas temperaturas de 60 e $70^{\circ} \mathrm{C}$.

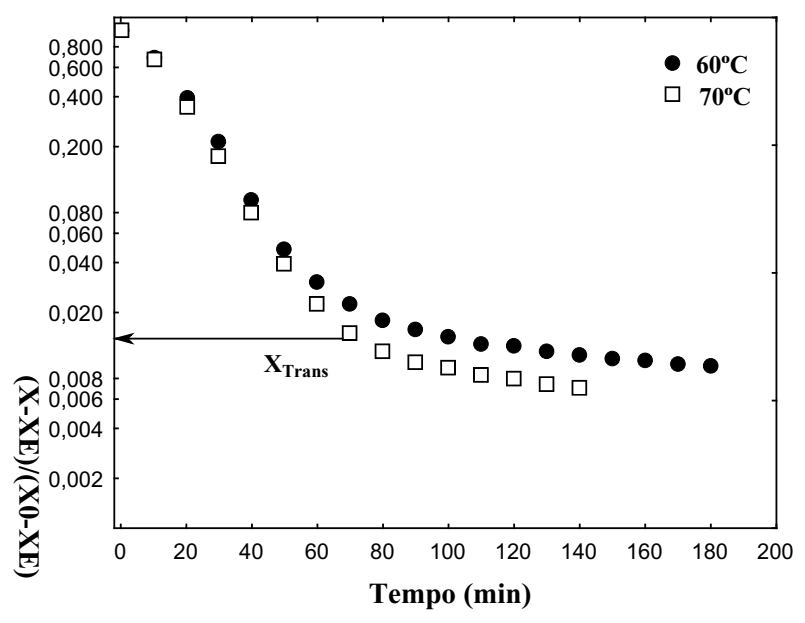

Figura 3: Curva do adimensional de água livre em função do tempo, $60^{\circ} \mathrm{C}$ e $70^{\circ} \mathrm{C}$

$\mathrm{Na}$ Figura 3 pode-se observar que o período de taxa decrescente controlou praticamente toda a operação, sendo constituído por duas fases. Os valores da umidade de transição do sólido $\left(\mathrm{X}_{\text {Trans }}\right)$ da primeira para a segunda fase foram de aproximadamente 0,15 (b.s.). Os valores do tempo total de secagem para alcançar a umidade comercial da quitosana (5 a 7\%, b.s.), nas duas condições analisadas, conforme Figura 3 , foram de $180 \mathrm{~min}$ para $60^{\circ} \mathrm{C}$ e $140 \mathrm{~min}$ para $70^{\circ} \mathrm{C}$. Na Tabela 1 são apresentados os valores da produção para cada temperatura de secagem utilizada, sendo estes obtidos pela razão entre a carga de sólidos da bandeja e o tempo total de secagem.

As curvas experimentais do adimensional de água livre (b.s) em função do tempo foram utilizadas para o cálculo dos valores da constante de secagem $\left(\mathrm{K}_{1,2}\right)$, através da Equação 7. A Tabela 1 apresenta os 
valores da constante de secagem da primeira $\left(\mathrm{K}_{1}\right)$ e da segunda fases $\left(\mathrm{K}_{2}\right)$ do período de taxa decrescente, bem como os valores do erro médio relativo $(\mathrm{E} \%)$ obtidos pela Equação 8 e do coeficientes de determinação $\left(\mathrm{R}^{2}\right)$.

Tabela 1: Valores da constante de secagem e da produção na secagem de quitosana em camada delgada nas temperaturas estudadas.

\begin{tabular}{|c|c|c|c|c|c|c|c|}
\hline $\begin{array}{c}\text { Temperatura } \\
\left({ }^{\circ} \mathrm{C}\right)\end{array}$ & $\begin{array}{l}\text { Produção } \\
\left(\mathrm{kg} / \mathrm{m}^{2} . \mathrm{h}\right)\end{array}$ & $\begin{array}{c}1^{\mathrm{a}} \text { fase } \\
\mathrm{K}_{1}\left(\min ^{-1}\right)^{*}\end{array}$ & $\begin{array}{l}E_{1} \\
(\%)\end{array}$ & $\left(\mathrm{R}^{2}\right)_{\mathrm{I}^{\mathrm{N}}}$ & $\begin{array}{c}2^{\mathrm{a}} \text { fase } \\
\mathrm{K}_{2}\left(\min ^{-1}\right)^{*}\end{array}$ & $\begin{array}{c}\mathrm{E}_{2} \\
(\%)\end{array}$ & $\left(\mathrm{R}^{2}\right)_{2^{*}}$ \\
\hline 60 & 1,33 & $0,074 \pm 0,001$ & 9,5 & 0,99 & $0,015 \pm 0,001$ & 6,7 & 0,97 \\
\hline 70 & 1,71 & $0,076 \pm 0,001$ & 9,9 & 0,99 & $0,015 \pm 0,001$ & 6,5 & 0,98 \\
\hline
\end{tabular}

*Valores médios \pm erros médios, obtidos em duplicada.

Analisando os dados da Tabela 1, pode-se observar que a temperatura influenciou na resposta da produção, aumentando em torno de $28 \%$ na temperatura de $70^{\circ} \mathrm{C}$ em relação à de $60^{\circ} \mathrm{C}$.

O bom ajuste da Equação 7 para o cálculo da constante de secagem $\mathrm{K}_{1,2}$, tanto para a primeira fase $\left(\mathrm{K}_{1}\right)$ quanto para a segunda fase $\left(\mathrm{K}_{2}\right)$ do período de taxa decrescente, pode ser evidenciado na Tabela 1 pelos altos valores apresentados do coeficiente de determinação $\left(\mathrm{R}^{2}\right)$ e pelos valores do erro médio relativo menores do que $10 \%$.

Os valores da constante de secagem $\left(\mathrm{K}_{1,2}\right)$ foram aproximadamente cinco vezes menores na segunda fase $\left(\mathrm{K}_{2}\right)$ em relação à primeira fase $\left(\mathrm{K}_{1}\right)$ do período de taxa decrescente, em ambas as temperaturas estudadas. Este fato demonstra que também a segunda fase $\left(\mathrm{K}_{2}\right)$ é importante no tempo total de secagem, apesar do material apresentar um baixo valor de umidade $\left(\mathrm{X}_{\text {trans }}=0,15, \mathrm{bs}\right)$ no início desta fase.

Os valores da viscosidade intrínseca das amostras de quitosana foram calculados através do método da viscosidade reduzida, pelo uso da equação de Huggins (Equação 3). A Figura 4 apresenta o ajuste linear dos valores da viscosidade reduzida em função da concentração da solução, para as amostras obtidas na temperatura de secagem de $60^{\circ} \mathrm{C}$. Nesta condição, o valor da viscosidade intrínseca foi aproximadamente de $145 \mathrm{~mL} \cdot \mathrm{g}^{-1}$, com um coeficiente de determinação $\left(\mathrm{R}^{2}\right)$ de 0,99 . Os valores da massa molecular das amostras de quitosana, calculados pela equação de Mark-Houwink-Sakurada (Equação 4), estão apresentados na Tabela 2.

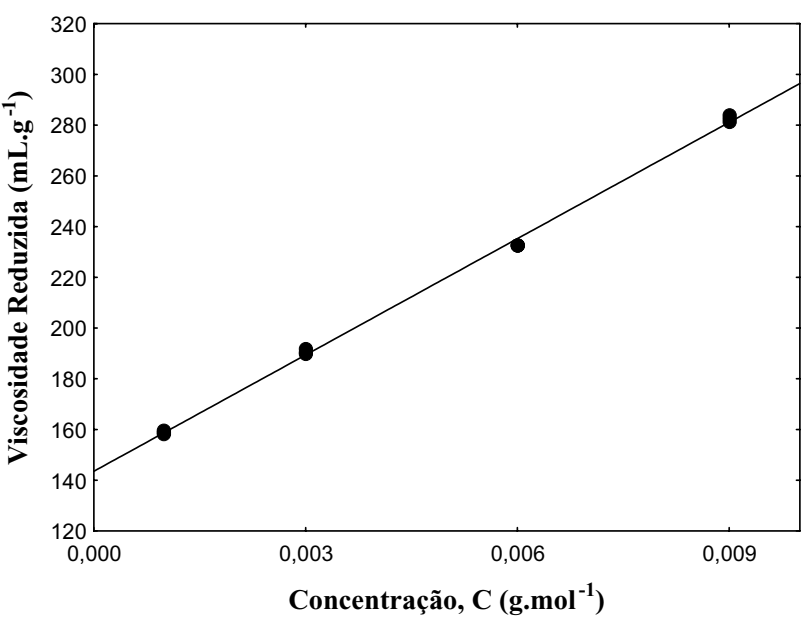

Figura 4: Curva da viscosidade reduzida em relação à concentração, secagem a $60^{\circ} \mathrm{C}$.

Utilizando-se a Equação 6, foram calculados os valores do grau de desacetilação $(\mathrm{GD} \%)$ para as amostras de quitosana, que estão apresentados na Tabela 2.

Foi realizado o teste de Tukey ao nível de confiança de $95 \%(p \leq 0,05)$ com os valores calculados para a massa molecular e para o grau de desacetilação.

Tabela 2: Valores obtidos da massa molecular e do grau de desacetilação da quitosana.

\begin{tabular}{c|c|c}
\hline $\begin{array}{c}\text { Temperatura } \\
\left({ }^{\circ} \mathrm{C}\right)\end{array}$ & $\begin{array}{c}\text { Massa Molecular, } \\
\mathrm{Mv}(\mathrm{kDa})^{*}\end{array}$ & $\begin{array}{c}\text { Grau de desacetilação, } \\
\text { GD }(\%)^{*}\end{array}$ \\
\hline In natura & $180 \pm 4,0^{\mathrm{a}}$ & $87 \pm 2,0^{\mathrm{a}}$ \\
\hline 60 & $205 \pm 6,0^{\mathrm{b}}$ & $86 \pm 1,0^{\mathrm{a}}$ \\
\hline 70 & $208 \pm 3,0^{\mathrm{b}}$ & $87 \pm 1,0^{\mathrm{a}}$ \\
\hline
\end{tabular}

*Valores médios \pm erros médios (em triplicata). Letras iguais $(\mathrm{p}>0,05)$, letras diferentes $(\mathrm{p} \leq 0,05)$.

Analisando os dados da Tabela 2, pode-se observar que as condições de secagem utilizadas alteraram significativamente a massa molecular do produto final ao nível de $95 \%(\mathrm{p} \leq 0,05)$. Esse aumento foi de aproximadamente $15 \%$ em relação ao valor da amostra inicial, e pode ser explicado devido à polimerização causada pela temperatura de secagem. Porém, nas duas temperaturas estudadas, os valores da massa molecular não apresentaram diferença significativa $(\mathrm{p}>0,05)$ pelo teste de Tukey. Na análise do grau de desacetilação, observa-se na Tabela 2 que as condições de secagem utilizadas não alteraram significativamente ao nível de $95 \%(\mathrm{p} \leq 0,05)$, 
recomendando-se a temperatura do ar de secagem de $70^{\circ} \mathrm{C}$ pelo fato desta condição ter apresentado a maior produção, como se observa na Tabela 1 .

\section{Conclusão}

A secagem de quitosana na forma de pellets cilíndricos, em camada delgada, com escoamento paralelo do ar, nas temperaturas de 60 e $70^{\circ} \mathrm{C}$, com carga de sólidos de $4 \mathrm{~kg} / \mathrm{m}^{2}$ e espessura das bandejas de $3 \mathrm{~mm}$, apresentou uma grande influência da temperatura do ar de secagem na produção de quitosana, obtendo-se um valor $28 \%$ superior à temperatura de $70^{\circ} \mathrm{C}$ em relação à de $60^{\circ} \mathrm{C}$.

As condições de secagem alteraram a massa molecular da quitosana significativamente $(p \leq 0,05)$ em relação à matéria-prima, porém as temperaturas de secagem utilizadas não afetaram a massa molecular do produto final de forma significativa, sendo os valores da massa molecular final, nas duas temperaturas estudadas, aproximadamente $15 \%$ superiores em relação aos valores da matéria inicial (180 kDa). O grau de desacetilação não foi afetado pelas condições de secagem, situando-se em torno de $87 \%$.

\section{Simbologia utilizada}

\begin{tabular}{c|c|c}
\hline $\mathrm{A}$ & Constante da Equação 4 & Adimensional \\
\hline $\mathrm{c}$ & Concentração & $\mathrm{g} / \mathrm{mL}$ \\
\hline $\mathrm{f}(\mathrm{x})$ & Valor correspondente ao & $\mathrm{mL}$ \\
\hline $\mathrm{GD}$ & volume de solução alcalina & $\%$ \\
\hline$\left[\mathrm{H}^{+}\right]$ & Concentração de íons positivos & Molar \\
\hline $\mathrm{k}$ & Constante da Equação 3 e 4 & Adimensional \\
\hline $\mathrm{K}$ & Constante de secagem & min ${ }^{-1}$ \\
\hline $\mathrm{Mv}$ & Massa Molecular & $\mathrm{kDa}$ \\
\hline $\mathrm{N}$ & Taxa de secagem & $\mathrm{kg}$ água/kgss.min \\
\hline $\mathrm{N}_{\mathrm{A}}$ & Concentração da solução ácida & Normal \\
\hline $\mathrm{N}_{\mathrm{B}}$ & Concentração da solução & Normal \\
\hline$[\mathrm{OH}-]$ & Concentração de íons & Molar \\
\hline $\mathrm{t}$ & negativos & min \\
\hline
\end{tabular}

\begin{tabular}{c|c|c}
\hline $\mathrm{V}$ & $\begin{array}{c}\text { Volume de solução alcalina adi- } \\
\text { cionada }\end{array}$ & $\mathrm{mL}$ \\
\hline $\mathrm{V}_{\mathrm{e}}$ & $\begin{array}{c}\text { Volume equivalente de solução } \\
\text { alcalina adicionada }\end{array}$ & $\mathrm{mL}$ \\
\hline $\mathrm{V}_{0}$ & Volume inicial da amostra & $\mathrm{mL}$ \\
\hline $\mathrm{X}$ & Umidade da amostra & $\mathrm{kg}$ água $/ \mathrm{kgss}$ \\
\hline $\mathrm{X}_{0}$ & Umidade inicial (base seca) & $\mathrm{kg}$ água $/ \mathrm{kgss}$ \\
\hline $\mathrm{X}_{\mathrm{C}}$ & Umidade crítica (base seca) & $\mathrm{kg}$ água $/ \mathrm{kgss}$ \\
\hline $\mathrm{X}_{\mathrm{E}}$ & Umidade de equilíbrio & $\mathrm{kg}$ água $/ \mathrm{kgss}$ \\
\hline $\mathrm{X}_{\text {Trans }}$ & Umidade de transição & $\mathrm{kg}$ água $/ \mathrm{kgss}$ \\
\hline $\mathrm{W}$ & Massa de amostra & $\mathrm{g}$ \\
\hline
\end{tabular}

\section{Letras gregas}

\begin{tabular}{c|c|c}
\hline $\mathrm{A}$ & $\begin{array}{c}\text { Constante da Equação 2 } \\
\text { Viscosidade dinâmica }\end{array}$ & $\begin{array}{c}\text { Adimensional } \\
\text { Poise }\end{array}$ \\
\hline$\eta$ & Viscosidade específica & Adimensional \\
\hline$\eta \mathrm{sp} / \mathrm{c}$ & Viscosidade reduzida & $\mathrm{mL} / \mathrm{g}$ \\
\hline$[\eta]$ & Viscosidade intrínseca & $\mathrm{mL} / \mathrm{g}$ \\
\hline$\rho$ & Densidade & $\mathrm{g} / \mathrm{mL}$ \\
\hline$v$ & Viscosidade cinemática & Stokes \\
\hline
\end{tabular}

\section{Referências}

ALSARRA, I. A. et al. Molecular weight and degree of deacetylation effects on lipase-loaded chitosan bead characteristics. Biomaterials, v. 23, p. 3637-3644, 2002.

ASSIS, O. B. G.; SILVA, V. L. Caracterização estrutural e da capacidade de absorção de água em filmes finos de quitosana processados em diversas concentrações. Polímeros: Ciência e Tecnologia, v. 13, n. 4, p. 223-228, 2003.

ASSOCIATION OF OFFICIAL ANALYTICAL CHEMISTS, A. O. A. C. Official Methods of Analysis. ed.14. Arligton, USA, 1995. v. 1.

BATISTA, L. M.; ROSA, C. A.; PINTO, L. A. A. Diffusive model with variable effetive diffusivity considering shrinkage in thin layer drying of chitosan. Journal Food Enginnering, v. 81, p. 127-132, 2007.

BOX, G. E. P.; HUNTER, W. G.; HUNTER, J. S. Statistics for experiments: an introduction to design, data analysis and model building. New York, John Wiley \& Sons, 1978. 
BRZESKI, M. M. Concept of chitin/chitosan isolation from antarctic krill (Euphasia superba Dana) shells on technical scale. Sea Fisheries Institute, Gdynia, Poland, 1982.

CHANG, K. L. B. et al. Heterogeneous N-deacetylation of chitin in alkaline solution.

Carbohydrate Research, v. 303, p. 327-332, 1997.

CHIRIFE, J. Fundamentals of the Drying Mechanism During Air Dehydration of Foods. In: MUJUMDAR, A. $\mathrm{S}$. Advances in drying. Washington. Ed. Hemisphere Publishing Corporation, v. 3, p. 73-102, 1983.

COMA, V. et al. Edible antimicrobial films based on chitosan matrix. Jounal of Food Science, v. 67, n. 3, p. 62-69, 2002.

CRAVEIRO, A. A.; CRAVEIRO A. C.; QUEIROZ D. C. Quitosana: a fibra do futuro, Parque de Desenvolvimento Tecnológico-PADETEC, Fortaleza-CE, 1999, p. 124.

GUPTA, K. C.; JABRIAL, F. H. Effects of degree of deacetylation and cross-linking on physical characteristics, swelling and release behavior of chitosan microspheres. Carbohydrate Polymers, v. 66, p. 43-54, 2006.

JIANG, X.; CHEN, L.; ZHONG, W. I. A new linear potentiometric titration method for the determination of deacetylation degree of chitosan. Carbohydrate Polymers, v. 54, p. 457-463, 2003.

REGE, P. R.; BLOCK, L. H. Chitosan processing: influence of process parameters during acidic and alkaline hydrolysis and effect of the processing sequence on the resultant chitosan's properties. Carbohydrate Research, v. 321, p. 235-245, 1999.

ROBERTS, A. F.; DOMSZY, J. G. Determination of the viscosimetric constants for chitosan. International Journal of Biological Macromolecules, v. 4, p. 374-377, 1982.

SILVA, H. S. R.; SANTOS, K. S. C. R.; FERREIRA, E. I. Quitosana: derivados hidrossolúveis, aplicações farmacêuticas e avanços. Química Nova, v. 29, n. 4, p. 776-785, 2006.

TAN, S. C.; et al. The degree of deacetylation of chitosan: advocating the first derivative UV-spectrophotometry method of determination. Talanta, v. 45, p. 713-719, 1998.

TOLAIMATE, A. et al. Contribution to the preparation of chitins and chitosans with controlled physico-chemical properties. Polymer, v. 44, n.26, 7939-7952, 2003.

WESKA, R. F. et al. Optimization of deacetylation in the production of chitosan from shrimp wastes: Use of response surface methodology. Journal of Food Engineering, v. 80, p. 749-753, 2007. 